\title{
OSCILLATIONS AND GLOBAL ATTRACTIVITY \\ IN A DISCRETE DELAY LOGISTIC MODEL
}

\author{
BY
}

\section{S. A. KURUKLIS AND G. LADAS}

The University of Rhode Island, Kingston, Rhode Island

Abstract. Consider the discrete delay logistic model

$$
N_{t+1}=\frac{\alpha N_{t}}{1+\beta N_{t-k}},
$$

where $\alpha \in(1, \infty), \beta \in(0, \infty)$, and $k \in \mathbb{N}=\{0,1,2, \ldots\}$. We obtain conditions for the oscillation and asymptotic stability of all positive solutions of Eq. (1) about its positive equilibrium $(\alpha-1) / \beta$. We prove that all positive solutions of Eq. (1) are bounded and that for $k=0$ and $k=1$ the positive equilibrium $(\alpha-1) / \beta$ is a global attractor.

1. Introduction and preliminaries. In her books $[8$, p. 22] and $[9$, p. 79], E. C. Pielou considered the delay difference equation

$$
N_{t+1}=\frac{\alpha N_{t}}{1+\beta N_{t-k}}
$$

where

$$
\alpha \in(1, \infty), \quad \beta \in(0, \infty), \quad \text { and } \quad k \in \mathbb{N}=\{0,1,2, \ldots\},
$$

as the discrete analogue of the delay logistic equation

$$
\dot{N}(t)=r N(t)\left[1-\frac{N(t-\tau)}{P}\right],
$$

where $r$ and $P$ are the growth rate and the carrying capacity of the population, respectively.

In this paper we obtain conditions for the oscillation and asymptotic stability of all positive solutions of Eq. (1) about its positive equilibrium $(\alpha-1) / \beta$. We prove that all positive solutions of Eq. (1) are bounded and that for $k=0$ and $k=1$ the positive equilibrium $(\alpha-1) / \beta$ is a global attractor.

One can derive Eq. (1) as follows: When $\tau=0$, the solution of Eq. (3) with $N(0)=N_{0}$ is given by

$$
N(t)=\frac{P}{1+\left(P / N_{0}-1\right) e^{-r t}} .
$$

Received February 16, 1990. 
An elementary calculation shows that

where $\alpha=e^{r}$ and $\beta=\left(e^{r}-1\right) / P$.

$$
N(t+1)=\frac{\alpha N(t)}{1+\beta N(t)},
$$

Pielou arrived at Eq. (1) assuming that there is a delay $k$ in the response of the growth rate per individual to density changes. This is also how one arrives at the delay logistic equation (3) from the logistic equation

$$
\dot{N}(t)=r N(t)\left[1-\frac{N(t)}{P}\right] \text {. }
$$

Pielou's interest in Eq. (1) was to show that "the tendency to oscillate is a property of the populations themselves and is independent of any extrinsic factors." That is, population sizes oscillate "even though the environment remains constant." According to Pielou, "oscillations can be set up in a population if its growth rate is governed by a density dependent mechanism and if there is a delay in the response of the growth rate to density changes. When this happens the size of the population alternatively overshoots and undershoots its equilibrium level."

The blow-fly, Lucilia cuprina, which was studied by Nicholson [7] is an example of a laboratory population that behaves in the manner described above.

Our aim in this paper is to study the oscillation and stability of all positive solutions of the delay difference equation

$$
x_{n+1}=\frac{\alpha x_{n}}{1+\beta x_{n-k}}, \quad n=0,1,2, \ldots .
$$

If $\alpha_{-k}, \ldots, \alpha_{0}$ are $(k+1)$ given constants, then Eq. (4) has a unique solution satisfying the initial conditions

$$
x_{i}=\alpha_{i} \quad \text { for } i=-k, \ldots, 0 .
$$

If the initial values are such that

$$
\alpha_{i} \geq 0 \quad \text { for } i=-k, \ldots,-1 \text { and } \alpha_{0}>0,
$$

then the unique solution of the IVP (4) and (5) is positive for $n \geq 0$.

In the sequel, we will only investigate solutions of Eq. (4) whose initial values satisfy condition (6).

A sequence $\left\{x_{n}\right\}$ is said to oscillate about zero or, simply, oscillate if the terms $x_{n}$ are not all eventually positive or eventually negative. A sequence $\left\{x_{n}\right\}$ is said to oscillate about $x^{*}$ if the sequence $\left\{x_{n}-x^{*}\right\}$ oscillates.

2. Necessary and sufficient conditions for oscillations. The main result in this section is the following theorem.

Theorem 1. Assume that (2) holds. Then every positive solution of Eq. (4) oscillates about its positive equilibrium $(\alpha-1) / \beta$ if and only if

$$
\frac{\alpha-1}{\alpha}>\frac{k^{k}}{(k+1)^{k+1}} \text {. }
$$

Before we can present the proof of Theorem 1 we need the following lemmas which are extracted from [2]. See also [3, and 4]. 
Lemma 1. Assume that $p \in(0, \infty)$ and $k \in\{1,2, \ldots\}$ and let $f$ be a real-valued continuous function such that

(i) $u f(u)>0$ for $u \neq 0$;

(ii) $\lim _{u \rightarrow 0}(f(u) / u)=1$;

(iii) there exists a $\delta>0$ such that

$$
\begin{aligned}
& \text { either } f(u) \leq u \text { for } 0<u<\delta, \\
& \text { or } \quad f(u) \geq u \text { for }-\delta<u<0 .
\end{aligned}
$$

Then every solution of the nonlinear difference equation

$$
x_{n+1}-x_{n}+p f\left(x_{n-k}\right)=0
$$

oscillates about 0 if and only if every solution of the linearized equation

$$
y_{n+1}-y_{n}+p y_{n-k}=0
$$

oscillates about 0 .

Lemma 2. Assume that $p \in(0, \infty)$ and $k \in\{1,2, \ldots\}$. Then every solution of

$$
y_{n+1}-y_{n}+p y_{n-k}=0
$$

oscillates about 0 if and only if

$$
p>\frac{k^{k}}{(k+1)^{k+1}} .
$$

Proof of Theorem 1. The change of variables

$$
x_{n}=\frac{\alpha-1}{\beta} e^{y_{n}}
$$

transforms Eq. (4) to the difference equation

$$
y_{n+1}-y_{n}+\frac{\alpha-1}{\alpha} f\left(y_{n-k}\right)=0, \quad n=0,1,2, \ldots,
$$

where

$$
f(u)=\frac{\alpha}{\alpha-1} \ln \frac{(\alpha-1) e^{u}+1}{\alpha} .
$$

Clearly, every solution of Eq. (8) oscillates about zero if and only if every solution of Eq. (4) oscillates about $(\alpha-1) / \beta$. One can easily see now that all the hypotheses of Lemma 1 are satisfied for the difference equation (8). In particular, note that (iii) is satisfied because

$$
f(u) \geq u \quad \text { for } u<0 .
$$

The linearized equation associated with Eq. $(8)$ is

$$
z_{n+1}-z_{n}+\frac{\alpha-1}{\alpha} z_{n-k}=0
$$

and, by Lemma 2, every solution of Eq. (9) oscillates if and only if (7) holds. The proof is now an elementary consequence of Lemmas 1 and 2. 
3. Boundedness and asymptotic stability. In this section we prove that all positive solutions of Eq. (4) are bounded. We also establish conditions for the asymptotic stability of the positive equilibrium of Eq. (4).

Theorem 2. Assume that $\alpha, \beta \in(0, \infty)$ and $k \in \mathbb{N}$. Then every solution $\left\{x_{n}\right\}$ of Eq. (4) whose initial values satisfy condition (6) is bounded and in fact

$$
x_{n} \leq \frac{\alpha^{k+1}}{\beta} \quad \text { for } n \geq k+1 .
$$

Proof. Clearly, $x_{n}>0$ for $n \geq 0$ and $x_{n+1} \leq \alpha x_{n}$ for $n \geq 0$. Hence, for $n \geq k$,

$$
x_{n+1}<\frac{\alpha x_{n}}{\beta x_{n-k}} \leq \frac{\alpha x_{n}}{\beta \frac{1}{\alpha} x_{n+1-k}} \leq \cdots \leq \frac{\alpha^{k+1}}{\beta}
$$

and the proof is complete.

Before we can discuss the asymptotic stability of the positive equilibrium of Eq. (4) we need the following result of Levin and May [5].

Lemma 3. Assume that $p \in \mathbb{R}$ and $k \in \mathbb{N}$. Then the difference equation

$$
x_{n+1}-x_{n}+p x_{n-k}=0, \quad n=0,1,2, \ldots,
$$

is asymptotically stable if and only if

$$
0<p<2 \cos \left(\frac{k \pi}{2 k+1}\right) \text {. }
$$

By using Lemma 3, it follows that the positive equilibrium of Eq. (4) is asymptotically stable if

$$
\frac{\alpha-1}{\alpha}<2 \cos \left\{\frac{k \pi}{2 k+1}\right\} \text {. }
$$

Note that condition (10) is satisfied for all $\alpha \in(1, \infty)$ if and only if $k=0$ or $k=1$. In each of these cases we will see in the next section that the positive equilibrium is a global attractor.

When $k=2,3, \ldots$ the positive equilibrium of Eq. (4) is asymptotically stable provided that

$$
1<\alpha<\frac{1}{1-2 \cos [k \pi /(2 k+1)]} .
$$

Observe that $1 /(1-2 \cos [k \pi /(2 k+1)])$ decreases to 1 as $k$ increases to $\infty$.

4. Global attractivity of the positive equilibrium. Our aim in this section is to establish a global attractivity result about the positive equilibrium $(\alpha-1) / \beta$, when the delay $k$ in Eq. (4) is equal to 1 . When $k=0$, the positive equilibrium $(\alpha-1) / \beta$ is known to be a global attractor. (For the proof of a more general result see [1].) In the special case of Eq. (4) with $k=0$, the following slightly stronger version than the result which follows from [1] is possible. See also May [6] where a similar result is stated without proof. 
Theorem 3. Assume that $\alpha \in(1, \infty)$ and $\beta \in(0, \infty)$. Then every solution of the difference equation

$$
x_{n+1}=\frac{\alpha x_{n}}{1+\beta x_{n}}, \quad n=0,1,2, \ldots,
$$

with $x_{0}>0$ converges monotonically to its positive equilibrium $(\alpha-1) / \beta$.

Proof. We consider three cases according to the initial value $x_{0}$.

Case 1. Assume that $x_{0}=(\alpha-1) / \beta$. Then it is easily seen by induction that

$$
x_{n}=(\alpha-1) / \beta \quad \text { for } n=0,1,2, \ldots \text {. }
$$

Case 2. Assume that $x_{0}>(\alpha-1) / \beta$. An easy inductive argument shows that

$$
x_{n}>(\alpha-1) / \beta \quad \text { for } n=0,1,2, \ldots \text {. }
$$

Next note that

$$
x_{n+1}-x_{n}=\frac{\alpha x_{n}}{1+\beta x_{n}}-x_{n}=\frac{(\alpha-1) / \beta-x_{n}}{1+\beta x_{n}} \beta x_{n}
$$

and thus $x_{n+1}-x_{n}<0$. Hence $\left\{x_{n}\right\}$ is monotonically decreasing and bounded below by $(\alpha-1) / \beta$. Thus $\left\{x_{n}\right\}$ has a limit not less than $(\alpha-1) / \beta$. Let $x$ be the limit. By taking the limits of both sides of Eq. (11) we see that either $x=(\alpha-1) / \beta$ or $x=0$. Therefore $\left\{x_{n}\right\}$ converges (decreasingly) to $(\alpha-1) / \beta$.

Case 3. Assume that $x_{0}<(\alpha-1) / \beta$. As in Case 2 , it is easily shown that $x_{n}<(\alpha-1) / \beta$. Also note that $x_{n+1}-x_{n}>0$. Thus $\left\{x_{n}\right\}$ has a limit which is equal to $(\alpha-1) / \beta$. Therefore $\left\{x_{n}\right\}$ converges (increasingly) to $(\alpha-1) / \beta$. The proof is complete.

The next theorem deals with the case $k=1$ and shows that, as in the case $k=0$, the positive equilibrium is a global attractor. However, unlike the case $k=0$, the convergence might not be monotonic.

Theorem 4. Assume that $a \in(1, \infty)$ and $\beta \in(0, \infty)$. Then every solution of the difference equation

$$
x_{n+1}=\frac{\alpha x_{n}}{1+\beta x_{n-1}}, \quad n=0,1,2, \ldots,
$$

with

$$
x_{-1} \geq 0 \text { and } \quad x_{0}>0
$$

converges to the positive equilibrium $(\alpha-1) / \beta$.

Proof. The change of variables

$$
x_{n}=\frac{\alpha-1}{\beta}+\frac{y_{n}}{\beta} \quad \text { for } n \geq-1
$$

transforms Eq. (13) to

$$
y_{n+1}=\frac{\alpha y_{n}-(\alpha-1) y_{n-1}}{\alpha+y_{n-1}}, \quad n=0,1,2, \ldots
$$

Furthermore, because of (13), the solutions of Eq. (12) are positive and so by (14)

$$
\alpha+y_{n}>1 \text { for } n \geq-1 \text {. }
$$


Note also that for $n \geq 0$

$$
\begin{gathered}
y_{n+1}=\frac{\alpha\left(y_{n}-y_{n-1}\right)+y_{n-1}}{\alpha+y_{n-1}}, \\
y_{n+1}-y_{n}=-\frac{(\alpha-1)+y_{n}}{\alpha+y_{n-1}} y_{n-1},
\end{gathered}
$$

and

$$
y_{n+2}=\frac{\alpha y_{n}}{\left(\alpha+y_{n-1}\right)\left(\alpha+y_{n}\right)}-\frac{(\alpha-1) y_{n-1}}{\alpha+y_{n-1}} .
$$

The proof will be complete if we show that

$$
\lim _{t \rightarrow \infty} y_{n}=0 \text {. }
$$

In view of (16), by using (18) we see that every solution of Eq. (15) which is eventually nonnegative or eventually nonpositive converges monotonically to zero.

Therefore, it remains to establish (20) for every solution of Eq. (15) which is "strictly" oscillatory, in the sense that it attains both positive and negative values. Such a solution consists of a "string" of consecutively negative terms followed by a string of consecutively nonnegative terms, etc. We will call these strings negative semicycles and positive semicycles.

By using (17) and (18) we see that every semicycle contains at least three terms and that the third term in a positive semicycle is positive. In fact if $y_{n-1}<0$ and $y_{n} \geq 0$, it follows from (16) and (18) that $y_{n+1}>y_{n} \geq 0$ and (16) and (19) imply that $y_{n+2}>0$. Also the maximum in a positive semicycle and the minimum in a negative semicycle is equal to the value of the second term in the respective semicycle. Let us prove it for a positive semicycle. The proof for a negative semicycle is similar and will be omitted. Indeed, as we saw above the second term in a positive semicycle is greater than the first. Finally, it follows from (18) that starting with the third term, all terms in a positive semicycle are decreasing.

Now to complete the proof, consider four consecutive semicycles $C_{r-1}, C_{r}, C_{r+1}$, $C_{r+2}$, with $C_{r-1}$ being a negative semicycle, as follows:

$$
\begin{array}{rlrl}
C_{r-1} & =\left\{y_{k+1}, y_{k+2}, \ldots, y_{l}\right\} & & \text { negative semicycle, } \\
C_{r} & =\left\{y_{l+1}, y_{l+2}, \ldots, y_{m}\right\} & & \text { positive semicycle, } \\
C_{r+1} & =\left\{y_{m+1}, y_{m+2}, \ldots, y_{n}\right\} & \text { negative semicycle } \\
C_{r+2} & =\left\{y_{n+1}, y_{n+2}, \ldots, y_{q}\right\} & & \text { positive semicycle. }
\end{array}
$$

We will establish the following two estimates from which the proof of (20) will become obvious:

$$
\left|y_{m+2}\right| \leqq \frac{(\alpha-1)^{2}}{\alpha^{2}-\alpha+1}\left|y_{k+2}\right|
$$

and

$$
y_{n+2} \leqq\left(\frac{\alpha-1}{\alpha}\right)^{2} y_{l+2}
$$


To this end, observe that from (19)

$$
y_{l+2} \leqq-\frac{(\alpha-1) y_{l-1}}{\alpha+y_{l-1}}
$$

and, since the function $x /(\alpha+x)$ is strictly increasing in $x$,

$$
y_{l+2} \leqq \frac{(\alpha-1)\left|y_{k+2}\right|}{\alpha-\left|y_{k+2}\right|} .
$$

Also, by using (19) and (23) we find

$$
\begin{aligned}
\left|y_{m+2}\right| & <\frac{(\alpha-1) y_{m-1}}{\alpha+y_{m-1}} \leqq \frac{(\alpha-1) y_{l+2}}{\alpha+y_{l+2}} \\
& \leqq \frac{(\alpha-1)(\alpha-1)\left|y_{k+2}\right| /\left(\alpha-\left|y_{k+2}\right|\right)}{\alpha+(\alpha-1)\left|y_{k+2}\right| /\left(\alpha-\left|y_{k+2}\right|\right)}=\frac{(\alpha-1)^{2}\left|y_{k+2}\right|}{\alpha^{2}-\left|y_{k+2}\right|} .
\end{aligned}
$$

As $\left|y_{k+2}\right|<\alpha-1$, it follows that

$$
\left|y_{m+2}\right| \leqq \frac{(\alpha-1)^{2}}{\alpha^{2}-\alpha+1}\left|y_{k+2}\right|
$$

and the proof of $(21)$ is complete.

In a similar way we see that

$$
y_{n+2} \leqq-\frac{(\alpha-1) y_{n-1}}{\alpha+y_{n-1}} \leqq \frac{(\alpha-1)\left|y_{m+2}\right|}{\alpha-\left|y_{m+2}\right|},
$$

and by using (24) and the increasing nature of the function $x /(\alpha+x)$ we find

$$
y_{n+2} \leq \frac{(\alpha-1)(\alpha-1) y_{l+2} /\left(\alpha+y_{l+2}\right)}{\alpha-(\alpha-1) y_{l+2} /\left(\alpha+y_{l+2}\right)}=\frac{(\alpha-1)^{2} y_{l+2}}{\alpha^{2}+y_{l+2}}<\frac{(\alpha-1)^{2}}{\alpha^{2}} y_{l+2},
$$

which proves (22) and completes the proof of the theorem.

\section{REFERENCES}

[1] L. Brand, A sequence defined by a difference equation, Amer. Math. Monthly 62, 489-492 (1955)

[2] I. Györi and G. Ladas, Oscillation Theory of Delay Difference Equations with Applications, Oxford Univ. Press, 1991

[3] I. Györi and G. Ladas, Linearized oscillations for equations with piecewise constant arguments, Differential Integral Equations J. 2, 123-131 (1989)

[4] G. Ladas, Recent developments in the oscillation of delay difference equations, Differential Equations: Stability and Control, Marcel Dekker, 1990

[5] S. Levin and R. May, A note on difference-delay equations, Theoret. Population Biol. 9, 178-187 (1976)

[6] R. M. May, Biological populations obeying difference equations: stable points, stable cycles, and chaos, J. Theoret. Biol. 51, 511-524 (1955)

[7] A. J. Nicholson, Compensatory reactions of populations to stresses and their evolutionary signifcance, Austral. J. Zool. 2, 9-65 (1954)

[8] E. C. Pielou, An Introduction to Mathematical Ecology, Wiley-Interscience, 1969

[9] E. C. Pielou, Population and Community Ecology, Gordon and Breach, New York, 1974 